\title{
Time-dependent failure criteria for polymer matrix composites: a review
}

\author{
Rui Miranda Guedes \\ Department of Mechanical Engineering, Faculty of Engineering (FEUP) of University \\ of Porto \\ Rua Dr. Roberto Frias s/n, 4200-465 Porto, Portugal \\ Email rmguedes@fe.up.pt
}

\begin{abstract}
This paper reviews several theoretical approaches for lifetime predictions proposed for polymer and polymer-based matrix composites. All these theories can be classified as global and homogenous approaches. Actually, regarding engineering applications, the global and homogeneous analyses are more convenient. Fracture mechanics, damage mechanics and energy-based failure criteria are presented and illustrated with published experimental data.
\end{abstract}

Keywords: Polymer-matrix composites (PMCs); Creep; Viscoelasticity, Strength; Durability

\section{INTRODUCTION}

In major cases the Strength Theories do not include creep to yield or creep to rupture process. Furthermore, since the stress-strain analysis is based on Continuum Mechanics it turns out to be a challenging task to predict failure in general and creep failure of polymer matrix based composites in particular. This can be surmounted by Fracture Mechanics or Damage Mechanics, which allow the inclusion of defects distribution into continuum models. Many approaches for the prediction of time dependent failures provide explicit elementary equations to predict lifetime. The examples given compare the theoretical lifetime predictions for two composite laminates against experimental data.

\section{ENERGY-BASED AND MAXIMUM STRAIN FAILURE CRITERIA}

These approaches conduct to direct relationships between modulus and strengths for viscoelastic materials, being the Reiner-Weissenberg $(\mathrm{R}-\mathrm{W})$ criterion [1] one of the first approaches. This criterion is based on the stored energy and on the limit for this energy, which is considered a material constant. The classical theory defines a limit 
value, considered a material property, for a stress or strain function and when this limit is exceeded failure takes place.

Theo et al. [2,3] and Boey et al. [4] presented an application of the R-W criterion to a three-element mechanical model, to describe the creep rupture of polymers. The predictions produced by such creep rupture model were in good agreement with the experimental data for several tested polymers. Raghavan and Meshii [5,6] developed a creep-rupture model, based on a creep model coupled with a critical stored energy criterion, and applied it to a epoxy and its carbon fiber reinforced composites with good results. These authors found out that the critical fracture energy was dependent on strain rate and temperature. However for elevated temperatures or very long times, a constant value for the critical fracture energy was considered suitable, in accordance with the R-W criterion.

Basically, the energy-based criteria are defined as functions of the free stored energy or the total stored energy. Accordingly $[7,8]$ it is possible to obtain the lifetime under constant load for each criteria, as a function of the applied load $\sigma_{0}$ and the strength under instantaneous conditions $\sigma_{R}$. Now, let us suppose that the unidirectional strain response of a linear viscoelastic material, under arbitrarily stress $\sigma(t)$, is given by the power law as,

$\varepsilon(t)=D_{0} \sigma(t)+D_{1} \int_{0}^{t}\left(\frac{t-\tau}{\tau_{0}}\right)^{n} \frac{\partial \sigma(\tau)}{\partial \tau} d \tau$,

where $D_{0}, D_{1}, n$ are material constants; and $\tau_{0}$ is time unity (equal to 1 second or 1 hour or 1day, etc.).

The definition for the total energy is given by

$W_{t}(t)=\int_{0}^{t} \sigma(\tau) \frac{\partial \varepsilon(\tau)}{\partial \tau} d \tau$

The free stored energy, using Hunter [9] formulation, is given by

$W_{s}(t)=\varepsilon(t) \sigma(t)-\frac{1}{2} D_{0} \sigma(t)^{2}-\frac{1}{2} \int_{0}^{t} \int_{0}^{t} D_{1}\left(\frac{2 t-\tau_{1}-\tau_{2}}{\tau_{0}}\right)^{n} \frac{\partial \sigma\left(\tau_{1}\right)}{\partial \tau_{1}} \frac{\partial \sigma\left(\tau_{2}\right)}{\partial \tau_{2}} d \tau_{1} d \tau_{2}$, (3)

Accordingly it is possible to obtain the lifetime under constant load for each criterion, as a function of the applied load $\sigma_{0}$ and the strength under instantaneous conditions $\sigma_{R}$ [7]. R-W Criterion (R-W) 


$$
\left(\frac{t_{f}}{\tau_{0}}\right)=\left(\frac{1}{2-2^{n}}\right)^{\frac{1}{n}}\left(\frac{D_{0}}{D_{1}}\right)^{\frac{1}{n}}\left(\frac{1}{\gamma}-1\right)^{\frac{1}{n}} .
$$

The Modified Reiner-Weissenberg (MR-W)

$$
\left(\frac{t_{f}}{\tau_{0}}\right)=\left(\frac{1}{2-2^{n}}\right)^{\frac{1}{n}}\left(\frac{D_{0}}{D_{1}}\right)^{\frac{1}{n}}\left(\frac{1}{\sqrt{\gamma}}-1\right)^{\frac{1}{n}} .
$$

Maximum Work Stress Criterion (MWS)

$$
\left(\frac{t_{f}}{\tau_{0}}\right)=\left(\frac{D_{0}}{D_{1}}\right)^{\frac{1}{n}}\left(\frac{1}{\gamma}-1\right)^{\frac{1}{n}} \text {. }
$$

Maximum Strain Criterion (MS)

$$
\left(\frac{t_{f}}{\tau_{0}}\right)=\left(\frac{D_{0}}{D_{1}}\right)^{\frac{1}{n}}\left(\frac{1}{\sqrt{\gamma}}-1\right)^{\frac{1}{n}} \text {. }
$$

where $\gamma=\sigma_{0}^{2} / \sigma_{R}^{2}$.

The practical application of these approaches is done using published experimental data $[10,11$ for two different composite laminates. One is a carbon fiber reinforced polymer, consisting of nine layers of plain woven cloth of carbon fiber and matrix vinylester resin (T300/VE) [10]. The other is the T800S/900-2B which consists of unidirectional T800 carbon fiber and 3900 epoxy resin with toughened interlayer [11] and with the following staking sequence $[45 / 0 / 90 /-45 / 90]_{\mathrm{s}}$. The experimental procedure $[10,11]$ was based on the Time-Temperature-Superposition Principle (TTSP) to accelerate the tests. In many cases the Time-Temperature Superposition Principle (TTSP) applied to creep compliance $D(t ; T)$ holds valid for static and creep strength $\sigma_{R}\left(t_{f} ; T\right)$ with the same shift factors $a_{T}(T)$,

$$
D\left(t ; T_{0}\right)=D\left(\frac{t}{a_{T}\left(T_{1}\right)} ; T_{1}\right) \Rightarrow \sigma_{R}\left(t_{f} ; T_{0}\right)=\sigma_{R}\left(\frac{t_{f}}{a_{T}\left(T_{1}\right)} ; T_{1}\right),
$$

where $T$ represents the temperature and $T_{0}$ the reference temperature. From this a reduced time to failure is defined as

$$
t_{f}^{\prime}=\frac{t_{f}}{a_{T}(T)}
$$


As an example the shift factors for the T300/VE composite were determined as [10],

$$
\ln \left(a_{T} ; T_{0}\right)=\frac{\Delta H}{G}\left(\frac{1}{T}-\frac{1}{T_{0}}\right),\left\{\begin{array}{l}
\Delta H=163 \mathrm{KJ} / \mathrm{mol}, T<90^{\circ} C \\
\Delta H=504 \mathrm{KJ} / \mathrm{mol}, T>90^{\circ} C
\end{array}\right\},
$$

where is $G$ the gas constant $8.314 \mathrm{E}-3 \mathrm{KJ} /(\mathrm{K} \mathrm{mol})$

In table I are presented the elastic and viscoelastic properties of composite laminates and in figure 1 are depicted the obtained results.

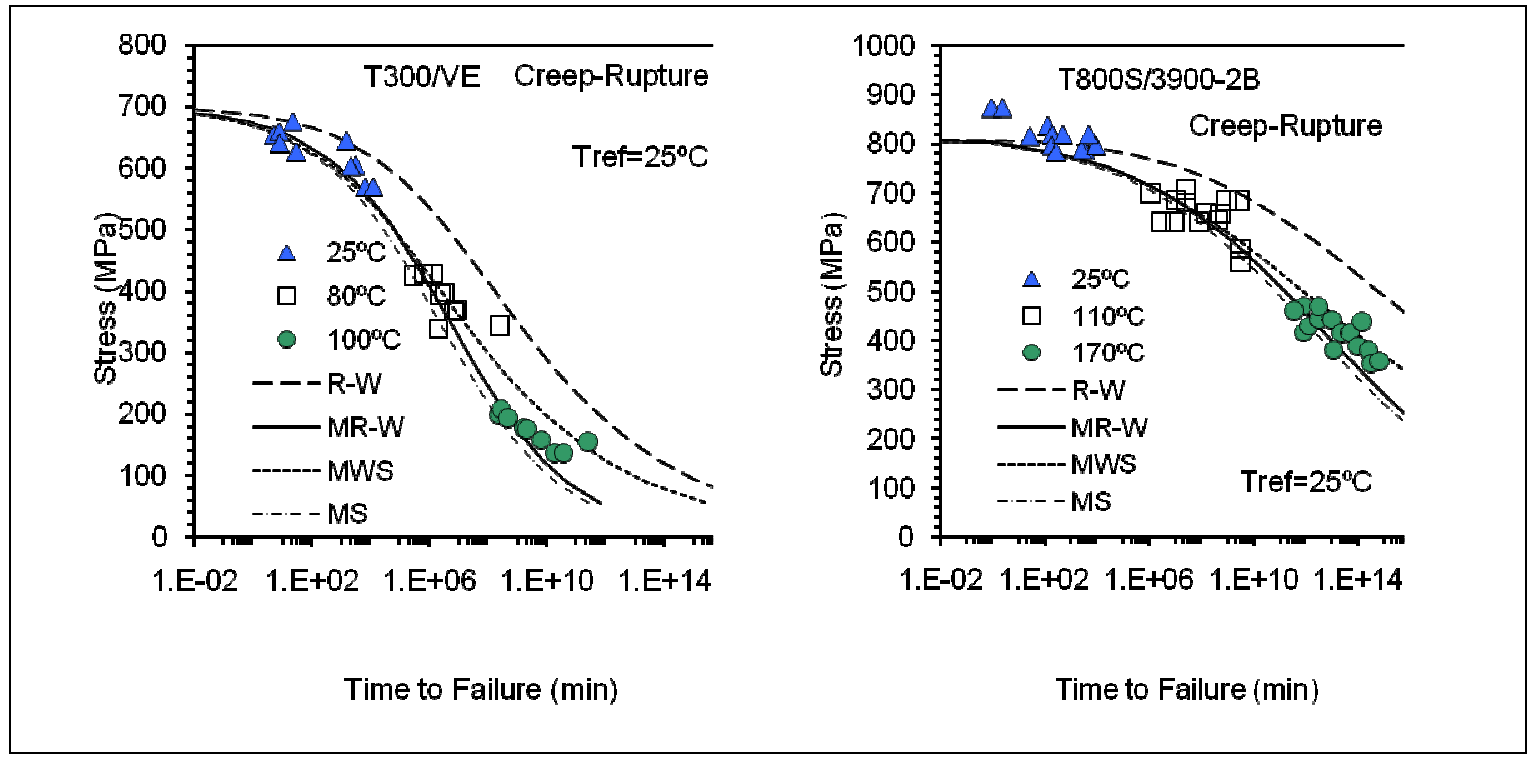

Figure 1 Theoretical lifetime curves based on energy based failure criteria compared against experimental data.

Table I Elastic and viscoelastic properties of composite laminates.

\begin{tabular}{|c|c|c|c|c|c|c|c|}
\hline \multirow{2}{*}{ Material } & \multirow{2}{*}{ Ref. } & \multirow{2}{*}{$\mathrm{T}\left({ }^{\circ} \mathrm{C}\right)$} & \multicolumn{5}{|c|}{$\begin{array}{l}\text { Instantaneous } \\
\text { Failure Stress } \\
(\mathrm{MPa})\end{array}$} \\
\hline & & & $D_{0}$ & \multirow{2}{*}{$\frac{D_{l}}{1.69 \mathrm{E}-05}$} & \multirow{2}{*}{$\frac{n}{0.209}$} & \multirow{2}{*}{$\frac{\tau_{0}}{1 \mathrm{~min}}$} & \multirow{2}{*}{$\begin{array}{c}\sigma_{R} \\
700\end{array}$} \\
\hline VE & [10] & $25^{\circ}$ & $3.60 \mathrm{E}-04$ & & & & \\
\hline T800S/3900-2B & [11] & $25^{\circ}$ & $1.85 \mathrm{E}-05$ & $6.33 \mathrm{E}-07$ & 0.119 & $1 \mathrm{~min}$ & 830 \\
\hline
\end{tabular}

\section{Criteria comparison}

If we consider the normalized rupture free energy at the rupture instant as

$$
\bar{w}_{R}=\frac{w_{R}}{\frac{D_{0}}{2} \sigma_{R}^{2}} .
$$


and the normalized applied stress as

$$
\bar{\sigma}_{0}=\frac{\sigma_{0}}{\sigma_{R}}
$$

Then it is possible to rewrite all the previous criteria in a non-dimensional way. The normalized free energies at rupture, function of the normalized applied stress, are obtained as follows.

The Reiner-Weissenberg criterion (R-W),

$\bar{w}_{R}=1$.

The Modified Reiner-Weissenberg criterion (MR-W),

$\bar{w}_{R}=\bar{\sigma}_{0}$.

The Maximum Stress Work criterion (MSW),

$\bar{w}_{R}=2^{n} \bar{\sigma}_{0}^{2}+\left(1-2^{n-1}\right)$.

The Maximum Strain criterion (MS),

$\bar{w}_{R}=\left(2^{n}-1\right) \bar{\sigma}_{0}^{2}+\left(2-2^{n}\right) \bar{\sigma}_{0}$.

The previous analysis is depicted in figure 2 for $n=0.3$. As one can observe the different criteria are similar within the region of higher stress level, i.e. higher than $50 \%$ of the stress rupture, except for the Reiner-Weissenberg criterion. This means that the lifetimes predicted by these criteria are very close for the higher stress levels, diverging for the lower stress levels. This is in accordance with lifetime predictions plotted in figure 1.

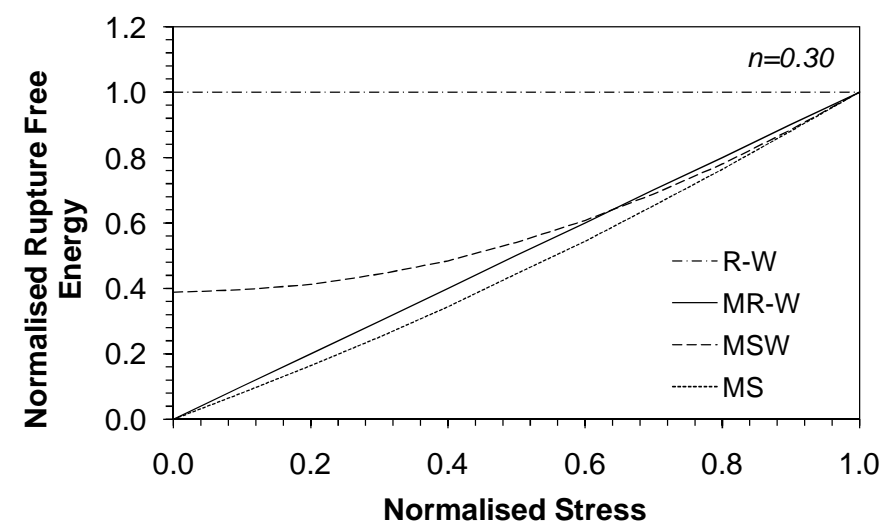

Figure 2 Normalized free energies at rupture versus normalized applied stress for different time-dependent failure criteria. 


\section{KINETIC RATE THEORY}

The rate theory of fracture is based on a molecular approach, i.e. on the kinetics of molecular flow and bond rupture of the polymer chains. Based on these approaches Zhurkov [12] presented on the first models to predict materials lifetime $t_{f}$ (except for very small stresses) in terms of a constant stress level $\sigma$,

$t_{f}=t_{0} \exp \left[\left(U_{0}-\gamma \sigma\right) / k T\right]$,

where $t_{0}$ is a constant on the order of the molecular oscillation period of $10^{-13} \mathrm{~s}, k$ is the Boltzmann constant, $T$ is the absolute temperature, $U_{0}$ is a constant for each material regardless its structure and treatment and $\gamma$ depends on the previous treatments of the material and varies over a wide range for different materials.

In table II are presented the material parameters for the Zhurkov criterion and in figure 3 are depicted the obtained results.

Table II Material parameters obtained for the Zhurkov criterion.

\begin{tabular}{cccc}
\hline Material & $\begin{array}{c}t_{0} \\
\min \end{array}$ & $U_{0}$ & $\gamma$ \\
& \multicolumn{2}{c}{} & \\
\hline VE & $1.667 \mathrm{E}-15$ & $2.628 \mathrm{E}-19$ & $1.634 \mathrm{E}-22$ \\
$\mathrm{~T} 800 \mathrm{~S} / 3900-2 \mathrm{~B}$ & $1.667 \mathrm{E}-15$ & $3.782 \mathrm{E}-19$ & $2.6062 \mathrm{E}-22$
\end{tabular}

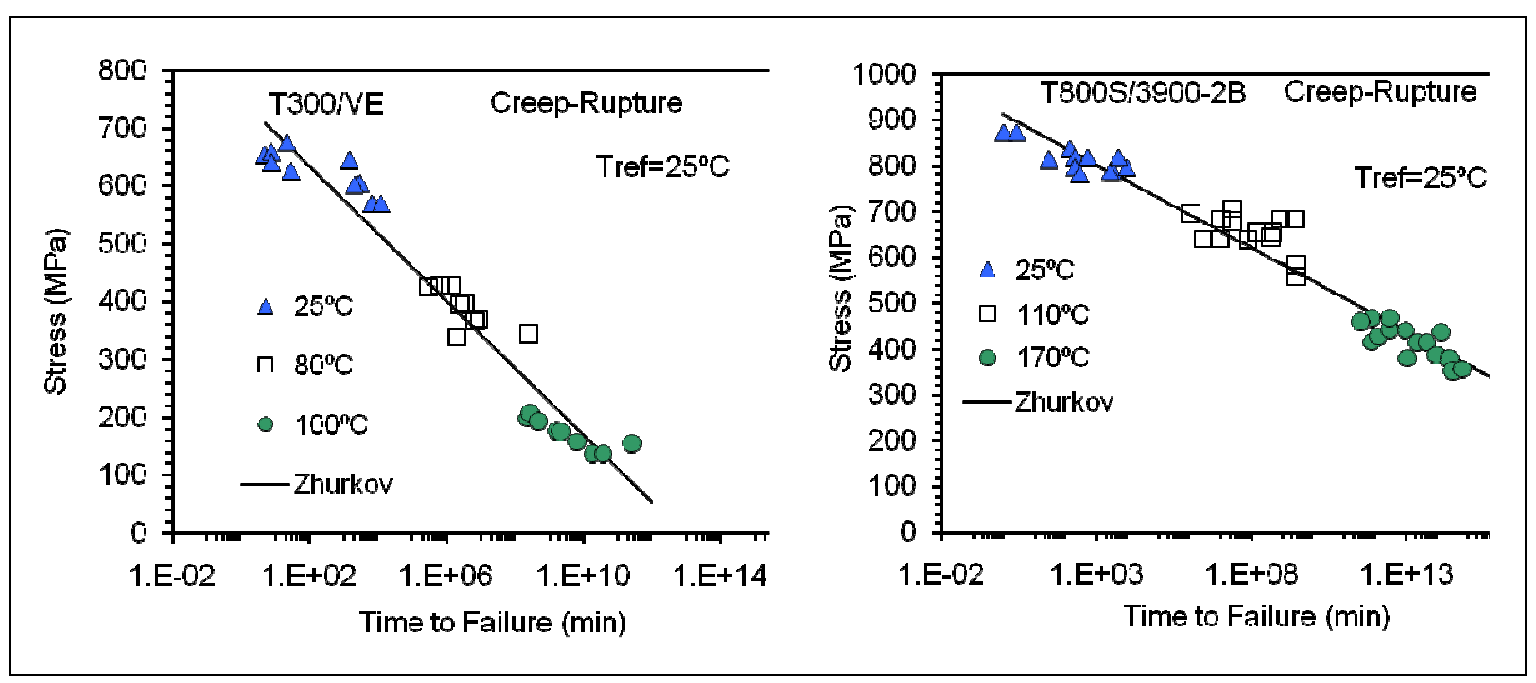

Figure 3 Theoretical lifetime curves based on Zhurkov criterion compared against experimental data. 


\section{FRACTURE MECHANICS EXTENDED TO VISCOELASTIC MATERIALS}

The fracture mechanics analysis was extended to viscoelastic media to predict the timedependent growth of flaws or cracks. Several authors developed extensive work on this area [13-18]. Schapery $[16,17]$ developed a theory of crack growth which was used to predict the crack speed and failure time for an elastomer under uniaxial and biaxial stress states [18]. For a centrally cracked viscoelastic plate with a creep compliance given by Equation (1) under constant load, Schapery [18] deduced, after some simplifications, a simple relation between stress and failure time,

$$
\left(\frac{t_{f}}{\tau_{0}}\right)=\left(B \sigma_{0}\right)^{-2(1+1 / n)},
$$

where $n$ is the exponent of the creep compliance power law and $B$ a parameter which depends on the geometry and properties of the material. Leon and Weitsman [19] and Corum et al. [20] used this approach where $B$ was considered an experimental constant, to fit creep-rupture data with considerable success. Recently Christensen [21] developed a kinetic crack formulation to predict the creep rupture lifetime for polymers. The lifetime was found from the time needed for an initial crack to grow to sufficiently large size as to cause instantaneous further propagation. The method assumed quasi-static conditions and only applies to the central crack problem. The polymeric material was taken to be in the glassy elastic state, as would be normal in most applications. For general stress, $\sigma(t)$ we have,

$$
1-\left(\sigma(t) / \sigma_{R}\right)^{1 / m}=\int_{0}^{t_{f} /\left(\alpha \tau_{0}\right)}\left(\sigma(\tau) / \sigma_{R}\right)^{1 / m+1} d \tau,
$$

where $\gamma=\sigma_{0}{ }^{2} / \sigma_{R}{ }^{2}, m$ is the exponent of the power law relaxation function and $\alpha$ is a parameter governed by the geometry and viscoelastic properties. For constant stress, $\sigma=\sigma_{0}$, the lifetime is given as,

$$
\left(\frac{t_{f}}{\tau_{0}}\right)=\frac{\alpha}{\sqrt{\gamma}}\left(\frac{1}{\sqrt{\gamma}^{1 / m}}-1\right) .
$$

In table III are presented the material parameters for the Schapery relationship and Christensen criterion and in figure 4 are depicted the obtained results. 
Table III Material parameters for the Schapery relationship and Christensen criterion.

\begin{tabular}{ccccc|ccc}
\hline \multirow{2}{*}{ Material } & \multicolumn{4}{c|}{ Schapery } & \multicolumn{3}{c}{ Christensen } \\
\cline { 2 - 8 } & \multirow{2}{*}{$B$} & $n$ & $-2(1+1 / \mathrm{n})$ & $t_{0}$ & $\sigma_{R}$ & $m$ & $\alpha$ \\
\hline VE & 0.000836 & 0.209 & -11.6 & $1 \mathrm{~min}$ & 690 & 0.100 & 1000 \\
T800S/3900-2B & 0.000992 & 0.119 & -34.0 & $1 \mathrm{~min}$ & 850 & 0.025 & 50 \\
\hline
\end{tabular}

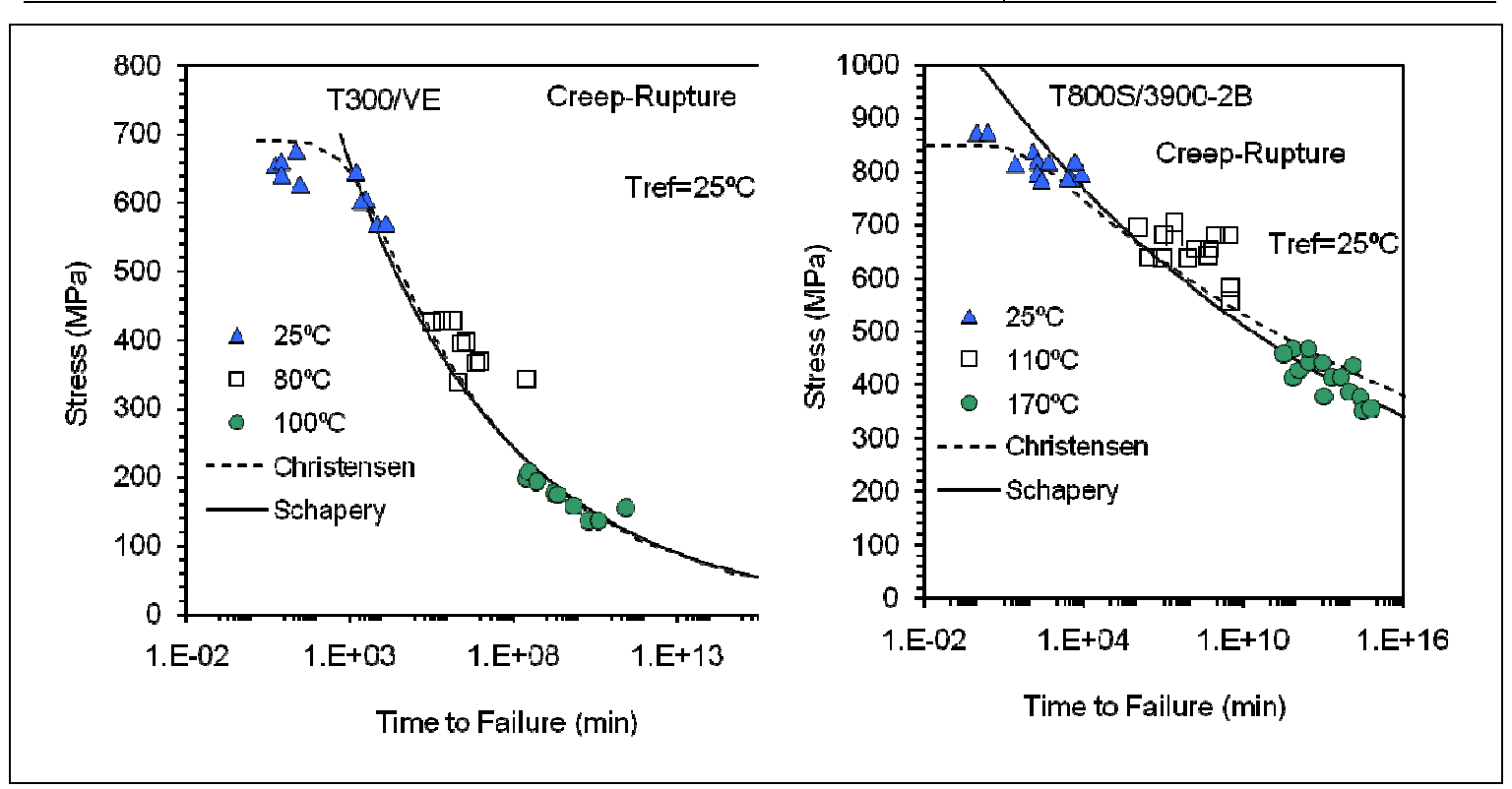

Figure 4 Theoretical lifetime curves based on Schapery and Christensen criteria compared against experimental data.

\section{CONTINUUM DAMAGE MECHANICS}

A classical approach to consider the degradation of mechanical properties is provided by the method of continuum damage mechanics (CDM). Following the original ideas of Kachanov [22], the net stress, defined as the remaining load bearing cross section of the material is given [23] by,

$$
\tilde{\sigma}=\frac{\sigma}{1-\omega},
$$

where $0 \leq \omega \leq 1$ is the damage variable. At rupture no load bearing area remains and the net stress tends to infinity when $\omega \rightarrow 1$.

Kachanov [22] assumes the following damage growth law

$$
\dot{\omega}(t)=C\left(\frac{\sigma(t)}{1-\omega(t)}\right)^{v},
$$


where $C$ and $v$ are material constants. This equation leads to a separable differential equation for $\omega(t)$, assuming $\omega(0)=0$

$$
(1-\omega(t))^{v} \dot{\omega}(t)=C \sigma^{v}(t) \Rightarrow 1-(1-\omega(t))^{1+v}=C(1+v) \int_{0}^{t} \sigma^{v}(\tau) d \tau
$$

The damage growth law is given as,

$$
\omega(t)=1-\left[1-C(1+v) \int_{0}^{t} \sigma^{v}(\tau) d \tau\right]^{\frac{1}{1+v}} .
$$

Assuming failure when $\omega=1$ then the following expression is obtained

$$
C(1+v) \int_{0}^{t} \sigma^{v}(\tau) d \tau=1
$$

From the previous relationship, the time to failure for creep is readily obtained assuming $\sigma(t)=\sigma_{0}$,

$t_{c}=\frac{1}{C(1+v) \sigma_{0}^{v}}$.

Clearly this result is equivalent to the obtained previously by using the Schapery theory. Therefore the creep lifetime expressions obtained for both theoretical approaches are directly comparable and are, in fact, equivalent, i.e.

$$
\left\{\begin{array} { r l } 
{ \frac { 1 } { C ( 1 + v ) } } & { = \frac { 1 } { \beta ^ { 2 ( 1 + 1 / n ) } } } \\
{ v } & { = 2 ( 1 + 1 / n ) }
\end{array} \Leftrightarrow \left\{\begin{array}{l}
\beta=[C(1+v)]^{\frac{1}{2(1+1 / n)}} \\
n=\frac{2}{v-2}
\end{array}\right.\right.
$$

even thought the parameters have distinct physical interpretations.

\section{THE CROCHET MODEL (TIME DEPENDENT YIELDING MODEL)}

A multi-axial yield/failure model for viscoelastic/plastic materials was developed by Naghdi and Murch [24] and later extended and refined by Crochet [25]. This approach was recently revised by Brinson [26]. In this theory, the total strain is assumed to be the sum of the viscoelastic and plastic strains. Stresses and strains are separated into elastic and viscoelastic deviatoric and dilatational components.

The yield function is given as

$$
f\left(\sigma_{i j}, \varepsilon_{i j}{ }^{p}, \chi_{i j}, \kappa_{i j}\right)=0 \text { and } \chi_{i j}=\chi_{i j}\left(\varepsilon_{i j}^{V}-\varepsilon_{i j}{ }^{E}\right) \text {, }
$$


Crochet gave specific form to the function $\chi_{\mathrm{ij}}$ such that

$\chi_{i j}=\sqrt{\left(\varepsilon_{i j}{ }^{V}-\varepsilon_{i j}{ }^{E}\right)\left(\varepsilon_{i j}{ }^{V}-\varepsilon_{i j}{ }^{E}\right)}$,

and defined a time dependent uniaxial yield function as (empirical equation)

$\sigma_{y}(t)=A+B e^{-C \chi}$,

where $\mathrm{A}, \mathrm{B}$ and $\mathrm{C}$ are material constants. No additional explanation for this empirical equation was given by Crochet. Assuming a linear viscoelastic law given by equation (1), the creep strain, under $\sigma(t)=\sigma_{0}$, can be calculated as

$\varepsilon(t)=D_{0} \sigma(t)+D_{1}\left(\frac{t}{\tau_{0}}\right)^{n} \sigma_{0}$,

The difference between viscoelastic and elastic strains in creep loading conditions becomes

$$
\left(\varepsilon_{11}^{V}-\varepsilon_{11}^{E}\right)^{2}=\left[D_{1}\left(\frac{t}{\tau_{0}}\right)^{n} \sigma_{0}\right]^{2}
$$

and the lateral strains become, upon assuming a constant Poisson's ratio $v$,

$$
\left(\varepsilon_{22}{ }^{V}-\varepsilon_{22}{ }^{E}\right)^{2}=\left(\varepsilon_{33}{ }^{V}-\varepsilon_{33}{ }^{E}\right)^{2}=v^{2}\left[D_{1}\left(\frac{t}{\tau_{0}}\right)^{n} \sigma_{0}\right]^{2},
$$

The time factor, $\chi$, in Crochet's time-dependent yield criteria for uniaxial tension now becomes,

$$
\chi=\sqrt{1+2 v^{2}}\left[D_{1}\left(\frac{t}{\tau_{0}}\right)^{n} \sigma_{0}\right]
$$

Using the previous formulations an equation for the time to yield, $t_{c}$, for a linear viscoelastic material can be found

$t_{c}=\left[\frac{1}{C \sigma_{f}} \frac{1}{D_{1} \sqrt{1+2 v^{2}}} \ln \left(\frac{B}{\sigma_{f}-A}\right)\right]^{1 / n}$,

where the symbol $\sigma_{f}$ is used, instead of $\sigma_{y}$, to indicate that the process may be used as well for creep rupture. 
In table IV are presented the material parameters for the Crochet lifetime equation and in figure 5 are depicted the obtained results.

Table III Material parameters for the Crochet lifetime equation.

\begin{tabular}{ccccccc}
\hline \multirow{2}{*}{ Material } & $\begin{array}{c}D_{l} \\
1 / \mathrm{MPa}\end{array}$ & $n$ & $v$ & $A$ & $B$ & $C$ \\
& & & $\mathrm{MPa}$ & $\mathrm{MPa}$ & \\
\hline VE & $1.692 \mathrm{E}-05$ & 0.2090 & 0.30 & 20 & 700 & 4 \\
T800S/3900-2B & $6.331 \mathrm{E}-07$ & 0.1185 & 0.30 & 10 & 900 & 70 \\
\hline
\end{tabular}

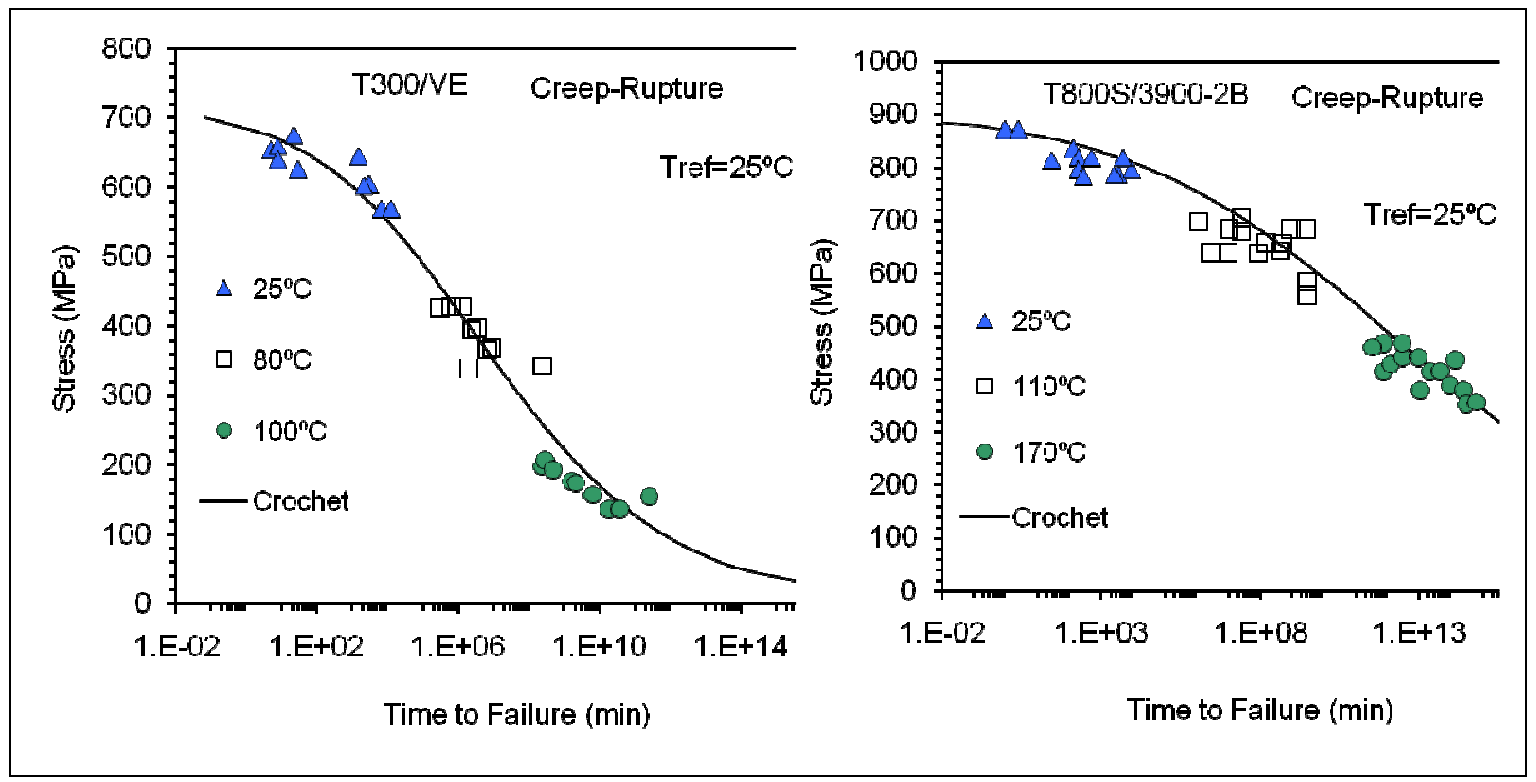

Figure 5 Theoretical lifetime curves based on Crochet criterion compared against experimental data.

\section{DISCUSSION AND CLOSURE}

Several time-dependent failure criteria applied to polymers and polymer-based matrix composites were present and illustrated using experimental data of two different composites. All criteria, excluding the energy-based failure criteria and the MS criterion, must have some or all the parameters obtained by curve-fitting the experimental lifetime data. This happens because the background theories lead to relative simple expressions recurring to empirical laws and simplifications, which ultimately enclose several variables related to the basic properties, with physical meaning, into few parameters. Consequently it becomes impossible to determine these parameters based on the basic properties, i.e. geometry, elastic and viscoelastic 
parameters and instantaneous strength. However, in this form, these lifetime expressions possess a more general character than it was initially devised.

One important characteristic of these criteria it is their forecast lifetime capability, based on short-time experimental data. In that respect all the present approaches appear to possess this capability. This review also shows the remarkable ability of energy-based failure and MS criteria to forecast lifetime based on viscoelastic parameters and instantaneous strength.

\section{ACKNOWLEDGEMENTS}

The research hereby presented was partially supported by Fundação para a Ciência e Tecnologia (Ministério da Ciência e do Ensino Superior) through the project PTDC/EME/67791/2006.

\section{References}

1. Reiner M, Weissenberg K., 'A thermodynamic theory of the strength of the materials', Rheol Leaflet 1939;10(1):12-20.

2. Teoh SH, Cherry BW, Kaush HH., 'Creep rupture modelling of polymers', International Journal of Damage Mechanics 1992;1(2):245-56.

3. Theo SH, 'Computational aspects in creep rupture modelling of polypropylene using an energy failure criterion in conjunction with a mechanical model', Polymer 1990;31:2260-6.

4. Boey FYC, Teoh SH., 'Bending creep rupture analysis using a nonlinear criterion approach', Mater Sci Engng 1990;A123:13-19.

5. Raghavan J, Meshii M., 'Prediction of creep rupture of unidirectional carbon fiber reinforced polymer composite', Materials Science and Engineering A 1995;197(2):237-249.

6. Raghavan J, Meshii M., 'Creep rupture of polymer composites', Compos Sci Technol 1997a ;57:375-88.

7. Guedes RM., 'Mathematical Analysis of Energies for Viscoelastic Materials and Energy Based Failure Criteria for Creep Loading', Mechanics of TimeDependent Materials 2004;8: 169-192.

8. Guedes RM., 'Lifetime predictions of polymer matrix composites under constant or monotonic load', Composites: Part A 2006;37:703-715.

9. Hunter, S.C., 'Tentative equations for the propagation of stress, strain and temperature fields in viscoelastic solids', J. Mech. Phys, Solids 1961;9(1):39-51.

10. Miyano Y., Nakada M., Sekine N., 'Accelerated Testing for Long-term Durability of FRP Laminates for Marine Use', Journal of Composite Materials 2005;39(1):5-20.

11. Miyano Y, Nakada M, Nishigaki K, 'Prediction of long-term fatigue life of quasi-isotropic CFRP laminates for aircraft use', International Journal of Fatigue 2006;28 (10): 1217-1225.

12. Zhurkov, S.N., 'Kinetic Concept of the Strength of Solids', International Journal of Fracture 1984; 26(4): 295-307 
13. Knauss, W.G., 'Delayed failure-the griffith problem for linearly viscoelastic materials', Internat. J. Fracture 1970; 6:7-20.

14. Kostrov, B.V. and Nikitin, L.V., 'Some general problems of mechanics of brittle fracture', Arch. Mech. Stos. (English version) 1970; 22:749-776.

15. Christensen, R.M., 'Lifetime predictions for polymers and composites under constant load', J. Rheology 1981;25:517-528.

16. Schapery, R.A., 'Theory of crack initiation and growth in viscoelastic media. 1. Theoretical development', Internat. J. Fracture 1975;11(1):141-159.

17. Schapery RA., 'Theory of crack initiation and growth in viscoelastic media. 2. Approximate methods of analysis', Internat. J. Fracture 1975;11(3):369-388.

18. Schapery RA., 'Theory of crack initiation and growth in viscoelastic media. 3. Analysis of continuous growth', Internat. J. Fracture 1975;11(4):549-562.

19. Leon R., Weitsman YJ., 'Time-to-failure of randomly reinforced glass strand/urethane matrix composites: data, statistical analysis and theoretical prediction', Mechanics of Materials 2001;33(3):127-137.

20. Corum JM, Battiste RL, Ruggles MB, Ren W., 'Durability-based design criteria for a chopped-glass-fiber automotive structural composite', Composites Science and Technology 2001;61(8): 1083-1095.

21. Christensen RM., 'An evaluation of linear cumulative damage (Miner's Law) using kinetic crack growth theory', Mech Time-Dependent Mater 2002;6(4):363-77.

22. Kachanov, L. M., 'On the Time to Failure Under Creep Conditions', Izv. Akad. Nauk SSR, Otd. Tekhn. Nauk, 1958;8:26-31.

23. Stigh U., 'Continuum Damage Mechanics and the Life-Fraction Rule', Journal of Applied Mechanics, 2006;73(4):702-704.

24. Naghdi PM, Murch SA., 'On the mechanical behavior of viscoelastic/plastic solids', J Appl Mech September 1963;30:321-28.

25. Crochet MJ., 'Symmetric deformations of viscoelastic-plastic cylinders', J Appl Mech 1966;33:327-34.

26. Brinson H.F., 'Matrix dominated time dependent failure predictions in polymer matrix composites', Composite Structures 1999; 47:445-56. 